\title{
Evaluation of a predictive approach in steering the human locomotion via haptic feedback
}

This is the peer reviewed version of the following article:

Original:

Aggravi, M., Scheggi, S., Prattichizzo, D. (2015). Evaluation of a predictive approach in steering the human locomotion via haptic feedback. In Proc. 2015 IEEE/RSJ International Conference on Intelligent Robots and Systems (IROS 2015) (pp.597-602). New York : IEEE [10.1109/IROS.2015.7353433].

Availability:

This version is availablehttp://hdl.handle.net/11365/980703

since 2017-05-24T19:24:42Z

Publisher:

IEEE

Published:

DOI:10.1109/IROS.2015.7353433

Terms of use:

Open Access

The terms and conditions for the reuse of this version of the manuscript are specified in the publishing policy. Works made available under a Creative Commons license can be used according to the terms and conditions of said license.

For all terms of use and more information see the publisher's website.

(Article begins on next page) 


\title{
Evaluation of a predictive approach in steering the human locomotion via haptic feedback
}

\author{
Marco Aggravi $^{1}$, Stefano Scheggi ${ }^{1}$, and Domenico Prattichizzo ${ }^{1,2}$
}

\begin{abstract}
In this paper, we present a haptic guidance policy to steer the user along predefined paths, and we evaluate a predictive approach to compensate actuation delays that humans have when they are guided along a given trajectory via sensory stimuli. The proposed navigation policy exploits the nonholonomic nature of human locomotion in goal directed paths, which leads to a very simple guidance mechanism. The proposed method has been evaluated in a real scenario where seven human subjects were asked to walk along a set of predefined paths, and were guided via vibrotactile cues. Their poses as well as the related distances from the path have been recorded using an accurate optical tracking system. Results revealed that an average error of $0.24 \mathrm{~m}$ is achieved by using the proposed haptic policy, and that the predictive approach does not bring significant improvements to the path following problem for what concerns the distance error. On the contrary, the predictive approach achieved a definitely lower activation time of the haptic interfaces.
\end{abstract}

\section{INTRODUCTION}

Let us assume that a human wants to reach a final location in a large environment. Possible scenarios consist in helping a person who is in a dangerous situation or guiding a person toward a point of interest. In our approach, the human is free to select her/his desired linear velocity while control signals are sent to the human to steer her/his locomotion. Requirements of our approach are that the person should always remains in charge of the final decision to take, the type of correction provided to the user should be perceived as very soft, and unnatural stimulations must be avoided as much as possible.

A recent study [1] has shown a close relationship between the shape of human locomotor paths in goal-directed movements and the simplified kinematic model of a wheeled mobile robot. Thus, nonholonomic constraints similar to those of mobile robots seem to be at work when a human is walking. These results provided us with the theoretical ground for adapting control strategies developed for wheeled mobile robots, to human subjects. Although the aforementioned considerations were successful in guiding the users in mixed human-robot scenarios [2], [3], we decided to adapt and evaluate our previous researches to the path following problem, where the user has to follow a predefined trajectory relying on haptic cues.

The research leading to these results has received funding from the European Union Seventh Framework Programme FP7/2007-2013 under grant agreement n. 601165 of the project "WEARHAP - WEARable HAPtics for humans and robots" and from the European Union's Horizon 2020 research and innovation programme - Societal Challenge 1 (DG CONNECT/H) under grant agreement n. 643644 of the project "ACANTO: A CyberphysicAl social NeTwOrk using robot friends"

${ }^{1}$ Marco Aggravi, Stefano Scheggi, and Domenico Prattichizzo are with the Department of Information Engineering and Mathematics, University of Siena, Via Roma 56, I-53100 Siena, Italy.

${ }^{2}$ Domenico Prattichizzo is with the Department of Advanced Robotics, Istituto Italiano di Tecnologia, via Morego 30, I-16163 Genova, Italy

\{aggravi, scheggi, prattichizzo\}@dii.unisi.it
Moreover, from our previous works, we noticed that, when navigation cues are provided to users, they do not instantaneously actuate the given control input, i.e., there is a certain amount of delay between the time in which the stimulus is sent to the user and the instant in which the user transforms the provided signal in a motion behavior. In particular, we found an average response time of about $1 \mathrm{~s}$ when navigation cues are provided via vibrotactile stimuli. As a consequence, in this paper we also present a predictive approach to compensate the actuation delays that humans have when they are guided along a given trajectory via sensory stimuli (auditory, visual, and haptic), and we evaluate if such method can improve the human performance in guidance scenarios.

We validated the proposed method in a real scenario where human subjects were asked to walk along a set of predefined paths, whereas directional controls were sent to them via haptic cues. We chose to evaluate our guidance method through haptic interfaces since presenting the navigation information via the sense of touch is an appealing solution. In fact, visual and/or audio displays may be useless for people which are in dangerous situation with dense smoke or which are visually impaired. In search and rescue scenarios, background noise can make auditory feedback difficult to hear or understand. Haptic feedback has the advantage of being relatively uninterruptible since the operator is in direct physical contact with the tactile device [4].

Vibrotactile haptic guidance has been successfully exploited in the last years. Closely related to this work are the researches presented in [5], [6], [7], and [3]. In [5], a vibrotactile belt is used for waypoint navigation in an outdoor environment. A torso-mounted vibrotactile display was used in [6] to provide vibrotactile cues for improving the situational awareness of soldiers in a simulated building-clearing exercise. A vibrotactile belt was used in [7] where the authors presented a navigation guidance system that guides a human to a goal point. Differently from the aforementioned works, they modeled the human as a nonholonomic robot and showed that control algorithms used for mobile robots can be opportunely translated to human guidance scenarios. Strictly related is our previous work [3], where we presented a new paradigm for the assisted navigation of mixed humanrobot teams using haptic information. The user is blindfolded and guided along a predefined path assisted by a mobile robot while a vibrotactile feedback is provided via a haptic bracelet. Finally in [8], the authors proposed a mobile device for human navigation using multimodal communication (audio, visual, vibrotactile and directional skin-stretch stimuli).

The main source of inspiration for the predictive approach came from [9] where the authors developed a predictive haptic method based on a look-ahead algorithm. The algorithm was evaluated in virtual environment, where users were 


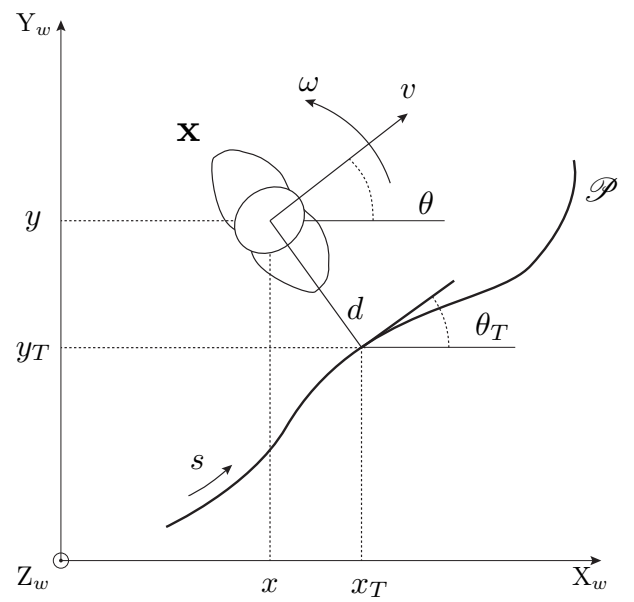

Fig. 1. Path following setup: $d$ represents the coordinate of the user position along the $y$-axis of the Frenet-Serret frame at $\left[x_{T}, y_{T}\right]^{T}$, i.e, the signed distance between the human and the orthogonal projection on to the path, $\theta_{T}$ and $\theta$ represent the angle between the $x$-axis of the world frame $\left\langle\mathrm{O}_{w}, \mathrm{X}_{w}, \mathrm{Y}_{w}, \mathrm{Z}_{w}\right\rangle$ and the $x$-axis of the Frenet-Serret and human frame, respectively.

asked to steer a vehicle along a path by using a customized grounded haptic interface.

The rest of the paper is organized as follows. Sect. II reviews the path following control strategy for mobile robots presented in [10], and shows how to adapt it in the proposed human guidance scenario. Sect. III presents our predictive approach to the actuation delay. In Sect. IV, we validate the proposed algorithms via real-world experiments, whereas in Sect. V, results are given and discussed. Eventually, in Sect. VI we summarize the main contributions of the paper, and we discuss possible avenues for future research.

\section{HumAN GUIDANCE VIA HAPTIC FEEDBACK}

In [1], authors found that nonholonomic constraints, similar to those describing the motion of mobile robots, seem to be at work when a human is walking. In particular, they showed that the human locomotion can be approximated by the motion of a unicycle system. An in-depth analysis revealed that the torso direction anticipates the trajectory direction of about $1 / 6 \mathrm{~s}$. In the rest of the paper, we model the human locomotion as a unicycle system. In what follows, we recall the path following problem, and we describe the vibrotactile policy used to steer the human along a predefined path.

\section{A. Path following problem}

Let us consider a human $\mathbf{x}(t)=[x(t), y(t), \theta(t)]^{T}$ whose kinematics can be abstracted as a unicycle model (see Fig. 1), where $[x(t), y(t)]^{T}$ and $\theta(t)$ represent the position and orientation of the human w.r.t. the world reference frame $\left\langle\mathrm{O}_{w}, \mathrm{X}_{w}, \mathrm{Y}_{w}, \mathrm{Z}_{w}\right\rangle$ at time $t \in \mathbb{R}_{>0}$, respectively. Let $v(t)$, $\omega(t)$ be the linear and angular velocities of the human, respectively. The human kinematics is described as

$$
\dot{\mathbf{x}}(t)=\left[\begin{array}{c}
\cos (\theta(t)) \\
\sin (\theta(t)) \\
0
\end{array}\right] v(t)+\left[\begin{array}{l}
0 \\
0 \\
1
\end{array}\right] \omega(t) .
$$

Let us consider the problem of steering the human locomotion in order to guide the user along a predefined path. This problem is referred to as path following problem. Let us briefly recall a common solution to it, [10].
Referring to Fig. 1, let $\mathscr{P}$ be a path to be followed which is parameterized by its arc length, $\left[x_{T}, y_{T}\right]^{T}$ be the orthogonal projection of the human on the path, $\theta_{T}$ be the orientation of the tangent to the path at $\left[x_{T}, y_{T}\right]^{T}$ w.r.t. $\mathrm{X}_{w}$ (the $x$-axis of the Frenet-Serret frame at $\left.\left[x_{T}, y_{T}\right]^{T}\right), d$ be the signed distance between $[x, y]^{T}$ and $\left[x_{T}, y_{T}\right]^{T}$, and $\tilde{\theta}=\theta-\theta_{T}$ be the heading of the human with respect to the path. Let $s \in \mathbb{R}$ be the curvilinear coordinate along the path, and $c(s)$ the curvature at that point, defined as $c(s)=d \theta_{T} / d s$.

The path $\mathscr{P}$ is chosen such that the radius of any tangent circle at two or more points of the path which does not contain any point of the curve is lower-bounded by some positive real number $r_{\text {min }}$, i.e., $|c(s)| \leq 1 / r_{\text {min }}, \forall s \in \mathbb{R}$.

With this parameterization, we can rewrite (1) as

$$
\begin{aligned}
\dot{s}(t) & =v(t) \cos (\tilde{\theta}(t))(1 /(1-c(s(t)) d(t))) \\
\dot{d}(t) & =v(t) \sin (\tilde{\theta}(t)) \\
\dot{\tilde{\theta}}(t) & =\omega(t)-v(t) \cos (\tilde{\theta}(t))(1 /(1-c(s(t)) d(t))) .
\end{aligned}
$$

Given $\mathscr{P}$ on the $\mathrm{X}_{w}-\mathrm{Y}_{w}$ plane, $v(t)$ bounded, $\dot{v}(t)$ bounded, the path following problem consists in finding a smooth feedback control law

$$
\omega(t)=\omega_{d}(t)=k(s(t), d(t), \tilde{\theta}(t), v(t))
$$

such that $\lim _{t \rightarrow \infty} d(t)=0$ and $\lim _{t \rightarrow \infty} \tilde{\theta}(t)=0$.

A linear feedback control law which stabilizes (2) is of the type

$$
\omega_{d}(t)=-k_{2} v(t) d(t)-k_{3}|v(t)| \tilde{\theta}(t)+a(t)
$$

where

$$
a(t)=v \cos (\tilde{\theta}(t)) \frac{c(s(t))}{1-c(s(t)) d(t)},
$$

is a corrective term coming from (2).

A nonlinear control approach for the same path following problem, holding asymptotical stability, is

$$
\omega_{d}(t)=-k_{2} d(t) v(t) \frac{\sin (\tilde{\theta}(t))}{\tilde{\theta}(t)}-k_{3}|v(t)| \tilde{\theta}(t)+a(t),
$$

being $k_{2}, k_{3} \in \mathbb{R}_{>0}, v \neq 0$, and $\lim _{t \rightarrow \infty} v(t) \neq 0$.

\section{B. Vibrotactile feedback and user response time}

In order to properly steer the user, we provide vibrotactile stimuli via two haptic bracelets [11]. Each bracelet is made by two motors with a vibration frequency range of 100$280 \mathrm{~Hz}$ (the maximal sensitivity is achieved around 200$300 \mathrm{~Hz}$, [12]), and typical normalized amplitude of $0.5 \mathrm{~g}$. In order to not overload the tactile channel and to not reduce the recognition time, we do not modulate the frequency of the signal, but we use a simple on/off mechanism, similar to [3]. Thus, an attractive haptic feedback mechanism is adopted: vibration of the left bracelet alerts the participant to turn left, and vice versa. When a bracelet is engaged, its motors alternatively vibrate for $0.2 \mathrm{~s}$ at a frequency of $250 \mathrm{~Hz}$.

Differently from [3], where a vibrotactile feedback was sent if the difference between the human angular velocity and the one computed from the controller was above a given threshold, in this work we set proper thresholds $d_{t h}$, $\theta_{t h} \in \mathbb{R}_{>0}$ on the position and orientation errors between the user and the path. Thus, if the absolute values of $d(t)$ and $\tilde{\theta}(t)$ are below the given thresholds, the angular velocity 
computed from the path following controller is zero. As a consequence, the proposed haptic feedback policy consists in sending a proper vibrotactile signal if the angular velocity $\omega_{d}(t)$ computed from the controller is not zero. In particular, the right bracelet is engaged if $\omega_{d}(t)<0$, while the left bracelet is activated if $\omega_{d}(t)>0$.

For evaluating the response time of the user to the vibrotactile stimuli, we tested the proposed haptic policy with seven healthy subjects (six males, age range 23-40, five right-handed). Participants were instructed to walk along a walkway, of about $4 \mathrm{~m}$, whilst wearing the bracelets and to react accordingly to the stimulus type (turn left or turn right), as soon as they perceived it. The stimulus was sent as soon as the users have walked for $1 \mathrm{~m}$. The bracelet continued to vibrate for $2 \mathrm{~s}$ after the activation. For each stimulus type, every subject performed 12 trials, organized in a pseudo-random order. All subjects were blindfolded and wore circumaural headphones, reproducing white noise to mask distracting ambient or cuing sounds from the bracelets. Human motion was tracked via an optical tracking system (Vicon Motion Systems Ltd, Oxford, UK).

The average time elapsed between the sending of the haptic stimulus and its actual perception by users, i.e., the start of their turning, was approximately $0.94 \mathrm{~s}$, with a standard deviation of $0.23 \mathrm{~s}$. We will use this information about the delay in our predictive approach and in our experimental validation, described in the following sections.

\section{A PREDICTIVE APPROACH FOR HUMAN GUIDANCE}

An assumption of our approach is that the human responds to vibrotactile haptic stimuli with a certain delay (see previous section). This means that the actuation of the angular input $\omega(t)$ in (1) is in fact delayed, i.e., $\omega(t-\delta)$, being $\delta \in \mathbb{R}_{>0}$ the response delay to the guiding stimulus.

Our purpose is to compute a proper control input $\omega_{d}(t)$, which will guide the user along the path when she/he will actuate it at time $t+\delta$. By considering the angular velocity actuation as $\omega(t-\delta)$ and by shifting the system (1) of $\delta$, we can rewrite the path following control in (3) as

$$
\begin{aligned}
\omega_{d}(t)=-k_{2} d(t+\delta) & v(t+\delta) \frac{\sin (\tilde{\theta}(t+\delta))}{\tilde{\theta}(t+\delta)} \\
& -k_{3}|v(t+\delta)| \tilde{\theta}(t+\delta)+a(t+\delta) .
\end{aligned}
$$

Thus, we need to compute a prediction of the user at time $t+\delta$ in order to compute the correct control input $\omega_{d}(t)$.

Due to the intrinsic discrete functioning of the computation machines, in what follows we switch from continuous time $t \in \mathbb{R}_{>0}$ to discrete time $k \in \mathbb{N}$. This means that we consider the actuation delay $\delta \in \mathbb{R}_{>0}$ as $\Delta \in \mathbb{N}$, which is a multiple of time step, and that we compute a desired angular velocity $\omega_{d, k}, \Delta$ steps in the future.

\section{A. Human tracking at time $k$}

Optical tracking systems are widely used for human tracking in indoor environment. Even tough these systems bring high precision and accuracy on the measurements, the acquired data are in general limited to the human position and orientation in space. In our approach, we have the necessity of a complete human state, i.e., human position, orientation, linear, and angular velocity, thus measurements from optical tracking systems are not enough. For obtaining such state, we exploited an Extended Kalman Filter (EKF) algorithm [13], so that we could use a reliable estimate of the human linear and angular velocities, together with its position and orientation. Let us consider a non-linear system, with the following dynamics

$$
\begin{aligned}
\mathbf{X}_{k+1} & =f_{k}\left(\mathbf{X}_{k}\right)+w_{k} \\
\mathbf{Y}_{k} & =h_{k}\left(\mathbf{X}_{k}\right)+v_{k}
\end{aligned}
$$

where $\mathbf{X}_{k} \in \mathbb{R}^{n}$ is the state of the system, $\mathbf{Y}_{k}$ are sensor measurements, $f_{k}\left(\mathbf{X}_{k}\right): \mathbb{R}^{n} \rightarrow \mathbb{R}^{n}$ is the state update, $h_{k}\left(\mathbf{X}_{k}\right): \mathbb{R}^{n} \rightarrow \mathbb{R}^{m}$ are measurements reading at step $k$, and $v_{k} \in \mathbb{R}^{n}, w_{k} \in \mathbb{R}^{m}$ are white Gaussian, independent processes with zero mean and covariance matrices $E\left[v_{k} v_{k}^{T}\right]=R_{k}$ and $E\left[w_{k} w_{k}^{T}\right]=Q_{k}$. The initial system condition $\mathbf{X}_{0}$ is considered as a Gaussian random vector, i.e., $\mathbf{X}_{0} \sim \mathcal{N}\left(\overline{\mathbf{X}}_{0}, P_{0}\right)$.

The EKF algorithm used for the our predictive approach is

$$
\begin{aligned}
\hat{\mathbf{X}}_{k+1}^{-} & =f_{k}\left(\hat{\mathbf{X}}_{k}^{+}\right), \\
P_{k+1}^{-} & =F_{k} P_{k}^{+} F_{k}^{T}+Q_{k},
\end{aligned}
$$

being

$$
\begin{aligned}
K_{k+1} & =P_{k+1}^{-} H_{k+1}^{T}\left(H_{k+1} P_{k+1}^{-} H_{k+1}^{T}+R_{k+1}\right)^{-1}, \\
\hat{\mathbf{X}}_{k+1}^{+} & =\hat{\mathbf{X}}_{k+1}^{-}+K_{k+1}\left(\mathbf{Y}_{k+1}-h_{k+1} \hat{\mathbf{X}}_{k+1}^{-}\right), \\
P_{k+1}^{+} & =\left(I-K_{k+1} H_{k+1}\right) P_{k+1}^{-},
\end{aligned}
$$

and

$$
F_{k}=(\nabla f)\left(\hat{\mathbf{X}}_{k}^{+}\right), \quad H_{k}=(\nabla h)\left(\hat{\mathbf{X}}_{k+1}^{-}\right),
$$

being the Jacobian matrices of $f(\cdot)$ and $h(\cdot)$, i.e, the linearization of the system dynamics and of the observation dynamics around $\mathbf{X}_{k}^{+}$and $\mathbf{X}_{k+1}^{-}$, respectively.

In our approach, the system state $\mathbf{X}_{k}$ is defined as $\mathbf{X}_{k}=$ $\left[\mathbf{x}_{k}^{T}, v_{k}, \omega_{k}\right]^{T}$, whereas the state evolution is based on the Euler integration method

$$
f_{k}\left(\mathbf{X}_{k+1}\right)=\left[\begin{array}{c}
x_{k+1} \\
y_{k+1} \\
\theta_{k+1} \\
v_{k+1} \\
\omega_{k+1}
\end{array}\right]=\left[\begin{array}{c}
x_{k}+v_{k} \cos \left(\theta_{k}\right) \Delta t \\
y_{k}+v_{k} \sin \left(\theta_{k}\right) \Delta t \\
\theta_{k}+\omega_{k} \Delta t \\
v_{k} \\
\omega_{k}
\end{array}\right]
$$

being $\Delta t=1 / f_{V}$ the discrete time step of the evolution of our system, and $f_{V}$ the sampling rate of the measurements acquisition system. Since the process noise $w_{k}$ is applied to the whole state, the human linear and angular velocity are modeled as "random walks".

The Jacobian matrix $F_{k}$ obtained by differentiating (1) w.r.t. to the state $\mathbf{X}_{k}$ has the form

$$
F_{k}=\left[\begin{array}{ccccc}
1 & 0 & -v_{k} \sin \left(\theta_{k}\right) \Delta t & v_{k} \cos \left(\theta_{k}\right) \Delta t & 0 \\
0 & 1 & v_{k} \cos \left(\theta_{k}\right) \Delta t & v_{k} \sin \left(\theta_{k}\right) \Delta t & 0 \\
0 & 0 & 1 & 0 & \Delta t \\
0 & 0 & 0 & 1 & 0 \\
0 & 0 & 0 & 0 & 1
\end{array}\right],
$$

while $H_{k}$ is set to be constant and equal to

$$
H=\left[\begin{array}{lllll}
1 & 0 & 0 & 0 & 0 \\
0 & 1 & 0 & 0 & 0 \\
0 & 0 & 1 & 0 & 0
\end{array}\right]
$$

The covariance matrices were chosen constant and not dependent on $k$, i.e., $R_{k}=R_{0}$ and $Q_{k}=Q_{0}$. 


\section{B. Human prediction at time $k+\Delta$}

Since the actuation delay introduced by the human is $\Delta$, we want to predict the human state $\Delta$ steps ahead from its current estimate at step $k$, i.e., $\mathbf{X}_{k+\Delta}^{-}=f_{k}^{p}\left(\mathbf{X}_{k}^{+}\right)$. For doing this, we consider a prediction step of the form

$$
f_{k}^{p}\left(\mathbf{X}_{k}^{+}\right)=\left[\begin{array}{c}
x_{k}+r_{k} \sin \left(\theta_{k+\Delta}\right)-r_{k} \sin \left(\theta_{k}\right) \\
y_{k}-r_{k} \cos \left(\theta_{k+\Delta}\right)+r_{k} \cos \left(\theta_{k}\right) \\
\theta_{k}+\omega_{k} \Delta \\
v_{k} \\
\omega_{k}
\end{array}\right],
$$

being $r_{k}=v_{k} / \omega_{k}$ the radius of the arc of circle routed by the human at step $k+\Delta$, and considering $v_{k}$ and $\omega_{k}$ constant between $k$ and $k+\Delta$.

In our predicting approach, we consider $\omega_{k}$ constant even if during $\Delta$ steps some angular velocity controls could be applied by the guidance system, resulting in changes in the actual value of $\omega_{k}$. However, in this preliminary work, we preferred to not model the variation of the angular velocity of the user when a haptic stimuli is perceived. In fact, from our evaluation on the activation delay (see Sect. II-B), we estimated a mean value for the amount of angular velocity variation applied by the human, together with its standard deviation. However, due to its high variability, we decided to not consider it. As a consequence, we modeled the linear and angular velocity evolution as a "random walk".

\section{EXPERIMENTAL VALIDATION}

In the previous sections, we described our approach for steering a human along a path by using haptic stimuli. We here present its evaluation, in which we tested four conditions:

- VN: the subject had no vision impairment (V), and the human state prediction $\Delta$ steps in the future was not enabled $(\mathrm{N})$;

- BN: the subject was blindfolded (B), and the human state prediction $\Delta$ steps in the future was not enabled $(\mathrm{N})$;

- VP: the subject had no vision impairment (V), and the human state prediction $\Delta$ steps in the future was enabled (P), using (6) for obtaining the control law $\omega_{d, k}$;

- BP: the subject was blindfolded (B), and the human state prediction $\Delta$ steps in the future was enabled (P), using (6) for obtaining the control law $\omega_{d, k}$;

In all modalities, participants were asked to walk along a pathway while directional cues concerning an ideal path to follow were sent to them in form of haptic stimuli (see Fig. 2). We tested our guidance system also with a blindfolded modality for having visibility situations similar to those frequently encountered in search and rescue scenarios. Participants were equipped with two vibrotactile bracelets, one per arm, and were allowed to choose their natural walking speed in order to perform the task. The human was modeled as a unicycle robot, and its state $\mathbf{X}_{k}$ was tracked with an EKF (4)-(5). Measurements $\mathbf{Y}_{k}$ corresponding to human poses were acquired with a optical tracking system (Vicon Motion Systems Ltd, Oxford, UK), composed of eight cameras. Participants worn eight passive retroreflective optical markers located on their torso, since there is a strong analogy between the steering wheels of a mobile robot and the human trunk [1]. The sampling frequency of the motion capture system was set to $f_{V}=100 \mathrm{~Hz}$. The four modalities were tested on five different paths (see Fig. 3, both columns): one linear path (first row), one path with low curvature (second row), one path with high curvature (fourth row), and their flipped versions (third and fifth rows).

The evaluation was performed on seven healthy subjects (five males, age range 19-65, four right-handed): two of them had great experience with the proposed vibrotactile bracelets; the remaining users had no experience with the vibrotactile interfaces. Two of them participated in the evaluation of the actuation delay (see Sect. II-B). None of the participants reported any deficiencies in perception abilities or physical impairments. The participants signed informed consent forms. All of them volunteered to the experiment, were informed about the purpose of the it, and were able to discontinue participation at any time. The trials were arranged in a pseudo-random order, whose list was the same for all the participants. Each participant performed 40 trials, i.e., four modalities repeated two times for each path, thus resulting in 280 collected trials (70 trials per modality).

For what regards the evaluation parameters, the distance and the orientation thresholds were chosen as $d_{t h}=0.15$ $\mathrm{m}$ and $\tilde{\theta}_{t h}=0.26 \mathrm{rad}=15 \mathrm{deg}$, respectively. The distance and orientation thresholds were chosen so that they would take in account natural oscillations of the human locomotion. The optical motion tracking frame rate $f_{V}$ was set to $100 \mathrm{~Hz}$, and the path following control for $\omega_{d, k}$ was computed every 20 steps (equivalent to $0.2 \mathrm{~s}$ ), according to the duration of the vibrotactile stimuli (see Sect. II-B). Concerning the EKF parameters, we chose $\sigma_{v}=0.05$, $\sigma_{w}=0.05, P_{0}=\mathbf{I}_{5}, R_{0}=\operatorname{diag}([0.01,0.01,0.03])$, and $Q_{0}=\operatorname{diag}([0.5,0.5,0.5,0.5,0.5])$, being $\mathbf{I}_{i}$ an identity matrix of dimension $i$. The initial configuration for the EKF state $\overline{\mathbf{X}}_{0}$ was chosen as the first point of the evaluated path (see Fig. 3). The controller gains were chosen as $k_{2}=10$ and $k_{3}=15$. Finally, for what regards the human state prediction, the number of steps to look in the future was set to 100 , i.e., $\Delta=100$, which leads to have a prediction of $1 \mathrm{~s}$ in the future.

\section{RESUlTS AND DiscusSiON}

Fig. 3 shows the ground truth paths and the trajectories performed by the users. Each row represents a different path. The first column reports the trajectories performed having the prediction of the human state disabled, while the second column depicts the trajectories performed using the predictive approach. For each trial $j$, the mean distance error from the path was computed as $e_{j}=\sum_{k=1}^{N}\left(\| \tilde{\mathbf{x}}_{k}-\right.$ $\left.\tilde{\mathbf{x}}_{k, T} \|\right) / N$, being $\tilde{\mathbf{x}}_{k}=\left[x_{k}, y_{k}\right]^{T}$ the position of the human on the $\mathrm{X}_{w}-\mathrm{Y}_{w}$ plane at step $k, \tilde{\mathbf{x}}_{k, T}=\left[x_{k, T}, y_{k, T}\right]^{T}$ its closest Frenet-Serret frame center at step $k, N$ the number of time steps the subject has taken to complete the trial, and $\left\|\tilde{\mathbf{x}}_{k}-\tilde{\mathbf{x}}_{k, T}\right\|$ the Euclidean norm between $\tilde{\mathbf{x}}_{k}$ and $\tilde{\mathbf{x}}_{k, T}$. For each modality, we computed the mean distance error $\bar{D}=\sum_{j=1}^{70} e_{j} / 70$. Similarly, we computed the mean linear velocity of the subject $\bar{V}$ as the sum of the means of the linear velocities during each trial over the number of trials, and the mean haptic stimuli $\bar{H}$, which is the percentage of time subjects were stimulated with haptic interfaces over the duration of the trials. These results are summarized in Fig. 4 and in Table I. 

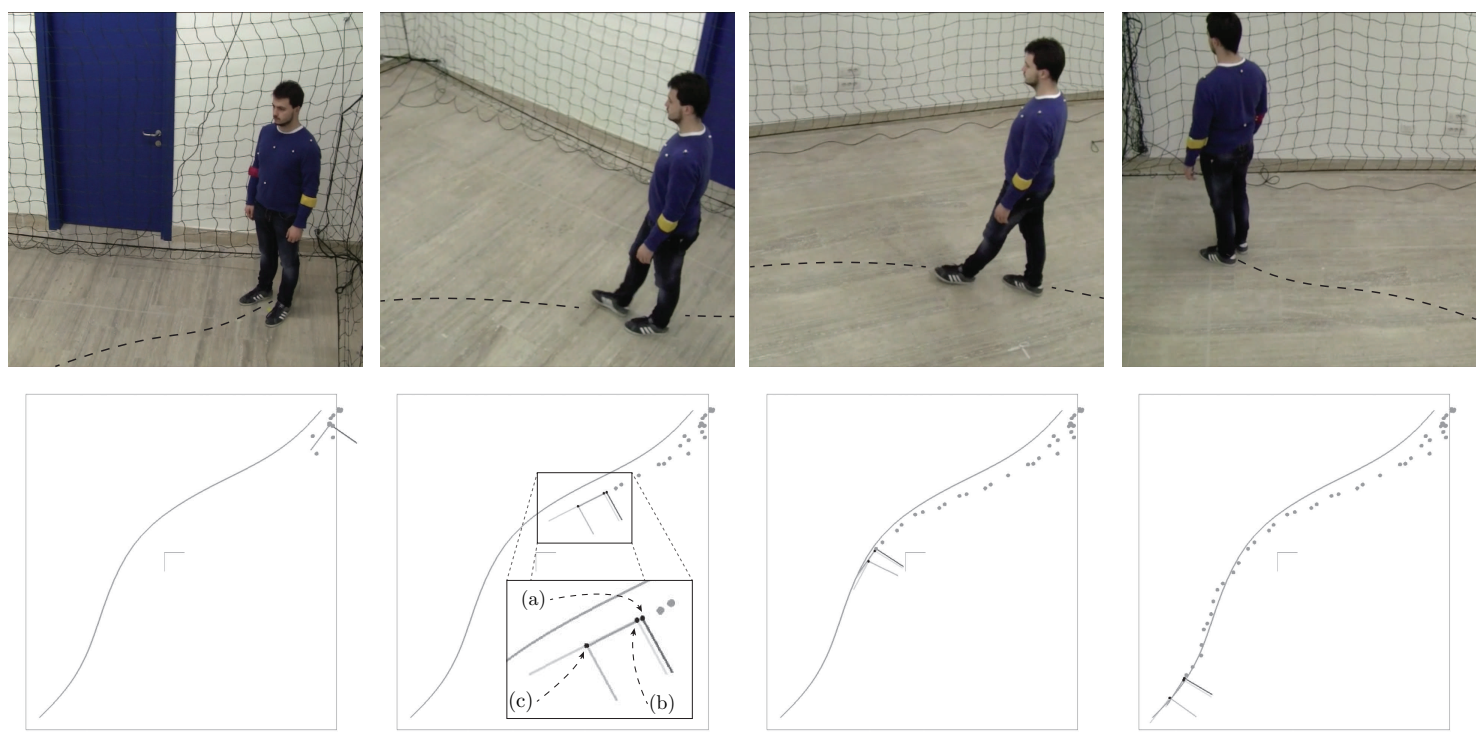

Fig. 2. Particular of a trial, VP modality: the subject is equipped with two vibrotactile bracelets, one per arm, and with eight passive retroreflective optical markers (visible in the upper row). The user is asked to walk at his natural walking speed following the haptic stimuli sent by the system. The subject is able to see the environment, but has no visual cue about the path (depicted for the reader in the upper figures). In the inlet, the human actual position (a), the EKF filtered state (b), and the predicted state (c) are shown.

\begin{tabular}{c|ccc} 
Modality & $\bar{D}[\mathrm{~m}]$ & $\bar{V}[\mathrm{~m} / \mathrm{s}]$ & $\bar{H}[\%]$ \\
\hline VN & $0.24( \pm 0.05)$ & $0.63( \pm 0.03)$ & $35( \pm 3.22)$ \\
BN & $0.23( \pm 0.06)$ & $0.59( \pm 0.02)$ & $34( \pm 3.97)$ \\
VP & $0.25( \pm 0.05)$ & $0.58( \pm 0.04)$ & $17( \pm 1.49)$ \\
BP & $0.24( \pm 0.04)$ & $0.48( \pm 0.02)$ & $17( \pm 1.67)$ \\
\hline
\end{tabular}

TABLE I

MEAN DistancE ERROR $\bar{D}$, MEAN HUMAN LINEAR VELOCITY $\bar{V}$, AND MEAN HAPTIC STIMULI ACTIVATION $\bar{H}$ (STANDARD DEVIATIONS ARE REPORTED INSIDE BRACKETS).

Comparisons of means among visual impairments (modality $\mathrm{V}$ vs. modality B) and among state prediction (modality $\mathrm{N}$ vs. modality $\mathrm{P}$ ) were tested using multiple repeatedmeasures ANalysis Of VAriance (ANOVA). In all conditions, collected data passed the Shapiro-Wilk normality test and the Mauchly's Test of Sphericity. A family-wise level $\alpha=0.05$ has been used for all tests.

From our results, we can say that the proposed predictive approach does not bring significant improvements to the path following problem for what concerns the distance error (see Fig. 4(a), first row), which was in mean around 0.24 m. An analog consideration can be made for the human linear velocity (see Fig. 4(a), second row), but in this case predicting the human state brought a significant reduction in the variability of the mean linear velocity itself, especially when the human was blindfolded, i.e., BN vs. BP modalities $(F(1,4)=68.23, p<0.05)$. For what regards the haptic activation time (see Fig. 4(b)), the proposed predictive approach brought improvements, since the activation time of the bracelets is significantly lower when the the predictive policy is used, VN vs. VP $(F(1,4)=974.00, p<0.05)$, and $\mathrm{BN}$ vs. BP modalities $(F(1,4)=277.93, p<0.05)$. Concerning the proposed haptic guidance, the obtained mean error of $0.24 \mathrm{~m}$ was deem acceptable for the application at hand, considering also the distance and orientation thresholds, which were set as $d_{t h}=0.15 \mathrm{~m}$ and $\tilde{\theta}_{t h}=15 \mathrm{deg}$. From the performed evaluation, the proposed predictive approach did not improve the performance of the haptic guidance system in terms of distance error from the path. One of the possible motivations is that in the proposed algorithm we considered a fixed delay in the user response time. Moreover, in the prediction step, we did not model the variation of the angular velocity $\omega_{k}$ due to the perception of the haptic stimuli by the user. Checking whether a dynamic delay and/or a variation of the angular velocity $\omega_{k}$ as input can improve the performance in terms of distance error is in the scope of future works.

\section{CONCLUSION}

In this paper, we presented a haptic guidance policy to steer the user along predefined paths, and we evaluated a predictive approach to compensate the actuation delays that humans have when they are guided along a given trajectory via sensory stimuli. We designed a vibrotactile policy which tries to be as intuitive as possible by exploiting the nonholonomic nature of human locomotion in goal directed paths. Results revealed the effectiveness of the proposed approach, showing an average error of $0.24 \mathrm{~m}$. From our results, we can say that the proposed predictive approach did not bring significant improvements to the path following problem for what concerns the distance error. On the contrary, it achieved a definitely lower activation time of the haptic interfaces. In future works, we will improve the predictive approach by estimating online the amount of delay the human needs to actuate the control input. Moreover, we will model the evolution of $\omega_{k}$ more accurately, by considering also variations of the angular velocity due to the perception of haptic stimuli.

\section{REFERENCES}

[1] G. Arechavaleta, J.-P. Laumond, H. Hicheur, and A. Berthoz, "On the nonholonomic nature of human locomotion," Autonomous Robots, vol. 25, no. 1-2, pp. 25-35, 2008. 

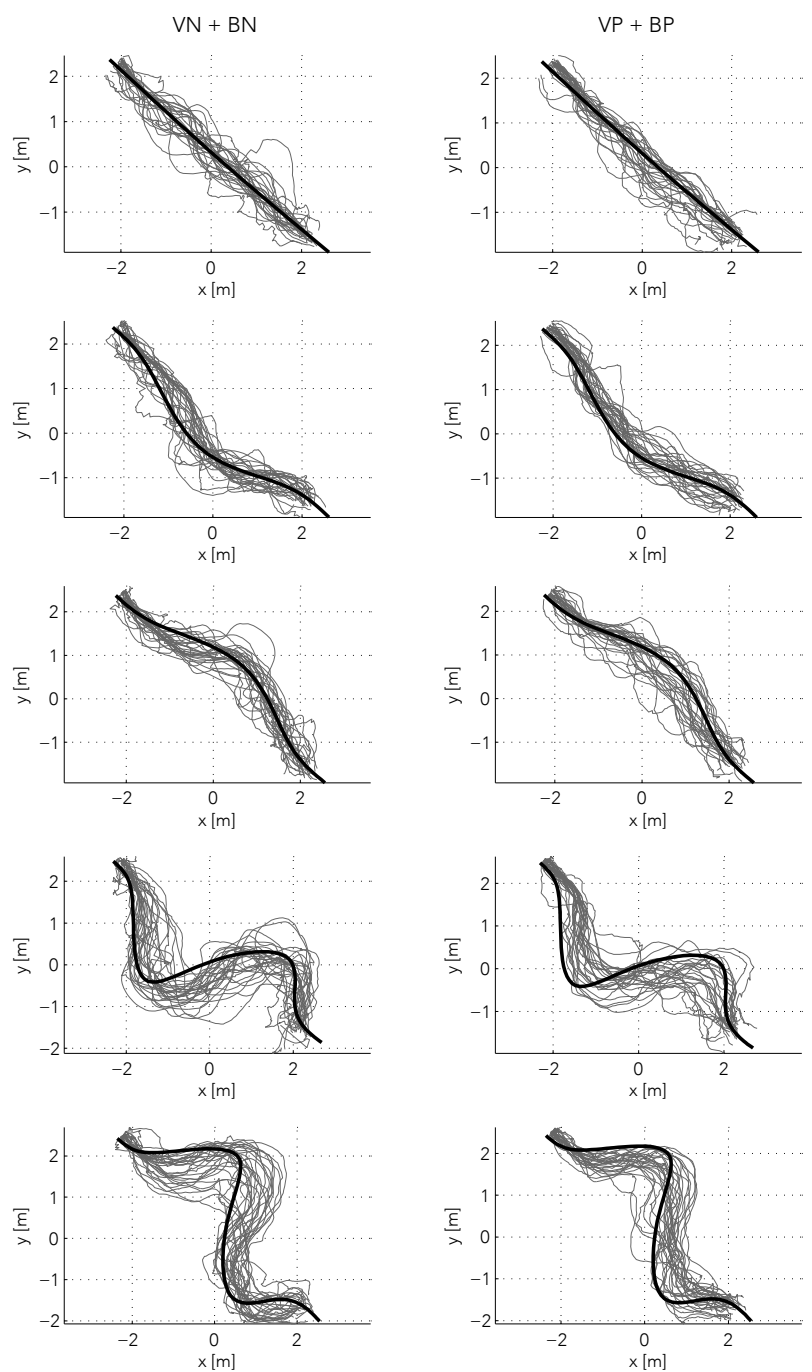

Fig. 3. Predifined path and collected user trajectories. From top to bottom, both columns: linear path, low curvature path, flipped version of the low curvature path, high curvature path, flipped version of the high curvature path. Each path is depicted with a black line, while the trajectories performed by the subjects are shown with grey lines. The left column shows the trajectories performed by the participants during the non predicting modalities, i.e., $\mathrm{VN}$ and $\mathrm{BN}$, while the right column shows the trajectories performed by the participants during the predicting modalities, i.e., VP and BP.

[2] S. Scheggi, F. Morbidi, and D. Prattichizzo, "Human-robot formation control via visual and vibrotactile haptic feedback," IEEE Transactions on Haptics, vol. 7, no. 4, pp. 499-511, 2014.

[3] S. Scheggi, M. Aggravi, F. Morbidi, and D. Prattichizzo, "Cooperative human-robot haptic navigation," in Proceedings IEEE International Conference on Robotics and Automation, ICRA. IEEE, 2014, pp. 2693-2698.

[4] J. Sibert, J. Cooper, C. Covington, A. Stefanovski, D. Thompson, and R. Lindeman, "Vibrotactile feedback for enhanced control of urban search and rescue robots," in Proceedings of the IEEE International Workshop on Safety, Security and Rescue Robotics, 2006.

[5] J. B. Van Erp, H. A. Van Veen, C. Jansen, and T. Dobbins, "Waypoint navigation with a vibrotactile waist belt," ACM Transactions on Applied Perception (TAP), vol. 2, no. 2, pp. 106-117, 2005.

[6] R. Lindeman, J. Sibert, R. Mendez-Mendez, S. Patil, and D. Phifer, "Effectiveness of directional vibrotactile cuing on a building-clearing task," in Proceedings of the SIGCHI conference on Human factors in computing systems. ACM, 2005, pp. 271-280.

[7] A. Cosgun, E. Sisbot, and H. Christensen, "Guidance for human navigation using a vibro-tactile belt interface and robot-like motion planning," in Proceedings IEEE International Conference on Robotics and Automation, ICRA. IEEE, 2014, pp. 6350-6355.

[8] R. L. Koslover, B. T. Gleeson, J. T. de Bever, and W. R. Provancher, "Mobile Navigation Using Haptic, Audio, and Visual Direction Cues
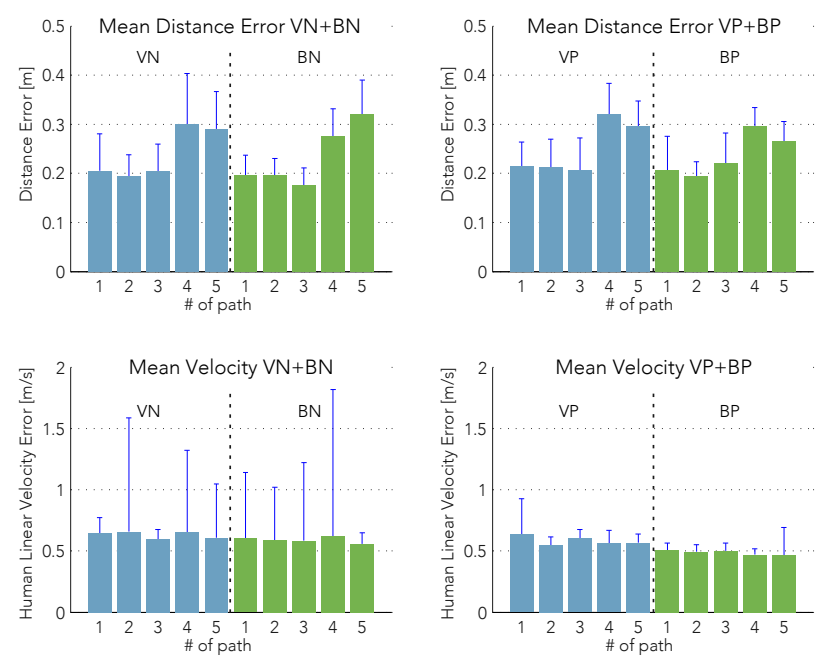

(a)
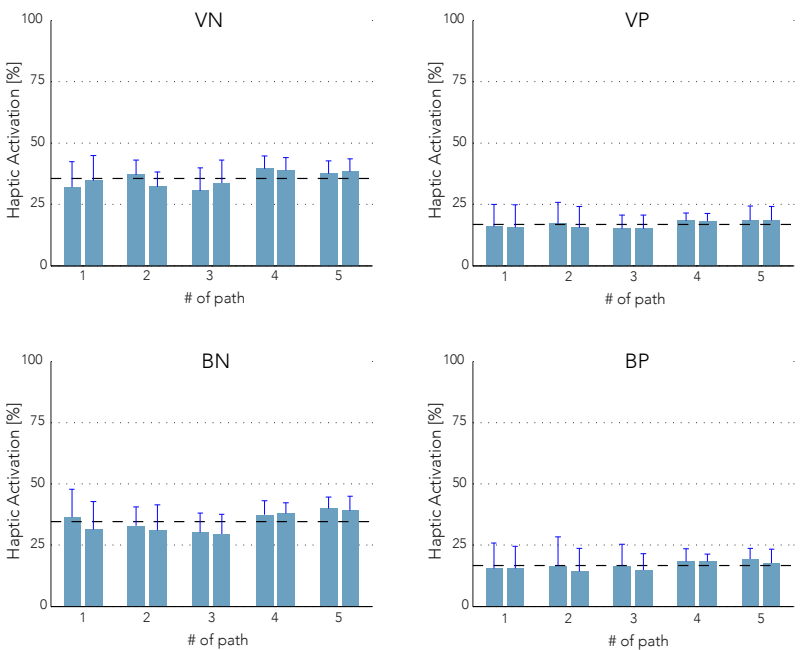

(b)

Fig. 4. Mean error distance and mean human linear velocity for the given paths (a) and mean haptic activation (b) grouped by vision condition and by predicting modalities (columns). Each bar blue vertical lines depict the standard deviation from the mean value, whereas for (b) overall means among the collected data are marked with black dashed lines.

with a Handheld Test Platform," IEEE Transactions on Haptics, vol. 5, no. 1, pp. 33-38, 2012.

[9] B. Forsyth and K. MacLean, "Predictive haptic guidance: intelligent user assistance for the control of dynamic tasks," Visualization and Computer Graphics, IEEE Transactions on, vol. 12, no. 1, pp. 103113, 2006.

[10] C. De Wit, G. Bastin, and B. Siciliano, Theory of robot control. Springer-Verlag New York, Inc, 1996.

[11] S. Scheggi, M. Aggravi, and D. Prattichizzo, "A vibrotactile bracelet to improve the navigation of older adults in large and crowded environments," in Proc. 20th IMEKO TC4 Int. Symp. and 18th Int. Workshop on ADC Modelling and Testing Research on Electric and Electronic Measurement for the Economic Upturn, Benevento, Italy, 2014.

[12] A. Riener, Sensor Actuator Supported Implicit Interaction in Driver Assistance Systems. Springer, 2010.

[13] P. S. Maybeck, Stochastic models, estimation, and control, ser. Mathematics in Science and Engineering, 1979, vol. 141. 